\title{
Modèles et conception publicitaire. Des pratiques incertaines, entre bricolage et braconnage
}

Arlette Bouzon

\section{OpenEdition}

1 Journals

Édition électronique

URL : http://journals.openedition.org/communicationorganisation/3460

DOI : 10.4000/communicationorganisation.3460

ISSN : $1775-3546$

Éditeur

Presses universitaires de Bordeaux

Édition imprimée

Date de publication : 1 octobre 2006

Pagination : 126-146

ISSN : 1168-5549

\section{Référence électronique}

Arlette Bouzon, « Modèles et conception publicitaire. Des pratiques incertaines, entre bricolage et braconnage », Communication et organisation [En ligne], 30 | 2006, mis en ligne le 21 juin 2012, consulté le 19 avril 2019. URL : http://journals.openedition.org/communicationorganisation/3460 DOI : 10.4000/communicationorganisation.3460

Ce document a été généré automatiquement le 19 avril 2019

(C) Presses universitaires de Bordeaux 


\title{
Modèles et conception publicitaire. Des pratiques incertaines, entre bricolage et braconnage
}

\author{
Arlette Bouzon
}

1 De l'équation gravitationnelle de Newton au simulateur complet d'un avion, un modèle est une construction de l'esprit (intellectuelle, matérielle ou logicielle) permettant de simuler les propriétés d'un domaine empirique. Faisant généralement l'objet d'une formalisation mathématique, déterministe ou stochastique (aléatoire), il permet de reproduire les mêmes effets (sorties) à partir des mêmes causes (entrées). A l'exception des modèles exclusivement reproductifs, "boîtes noires" fondés sur des réseaux de neurones par exemple, il bénéficie le plus souvent d'un pouvoir explicatif reposant sur une série d'hypothèses et la mise en place de concepts théoriques. Il suppose alors un certain niveau de compréhension des phénomènes physiques.

2 Sa validation passe par des batteries de tests comparatifs entre l'objet et son modèle, et diverses phases d'ajustement (réglage de ses paramètres). Le modèle est généralement conçu comme une aide à la décision, soit à titre de simple vérification (assurance), soit comme moyen d'optimisation (recherche de la meilleure décision). Un risque de confusion existe cependant entre le modèle et le phénomène étudié, notamment quand les théories sous-jacentes sont insuffisamment approfondies et ne traitent pas des situations particulières (modélisation simpliste telle que celle proposée par Newton avant qu'Einstein n'intervienne par exemple). De même, son pouvoir démonstratif apparent peut conduire à des détournements pour des usages abusifs, parfois fort éloignés de ceux de son contexte d'origine.

3 Qu'en est-il de la modélisation dans les sciences humaines et sociales (SHS) et particulièrement en communication organisationnelle? Nous tenterons d'apporter quelques éléments de réponse à cette question en nous interrogeant sur l'usage des modèles dans les pratiques publicitaires.

4 Longtemps considérée comme l'activité privilégiée du marketing, au point d'en être parfois confondue avec lui, la publicité cherche à obtenir un effet sur le comportement 
des individus d'une cible visée, en faveur d'un annonceur donné, l'activité publicitaire pouvant difficilement s'envisager indépendamment de ses retombées. Or l'interdépendance grandissante des marchés, l'essor des produits standardisés, l'émergence de médias internationaux et la diminution des budgets de communication accentuent depuis quelques années les exigences en matière de performance publicitaire, comme en témoignent les nombreux débats sur le neuromarketing qui agitent la profession depuis plusieurs mois.

5 Mais qu'elle soit quantitative ou qualitative, l'évaluation de la performance publicitaire reste une notion floue qui ne peut se confondre avec la simple augmentation des ventes. En effet, la publicité ne constitue qu'un moyen communicationnel parmi d'autres (les relations publiques, la promotion par exemple) dans un environnement saturé de messages concurrents et s'intègre dans une stratégie globale elle-même plus ou moins performante. En outre, cette mesure recouvre une pluralité de moyens (pré test en amont des campagnes, post test en aval, taux de mémorisation, de compréhension, d'agrément, d'attribution...) qui rendent difficilement compte du ressenti réel des prospects et consommateurs potentiels. Parallèlement, les discours transitent via de nouveaux médias, dont notamment Internet, qui intègrent de nouvelles règles de communication.

Dans ce contexte difficile, les publicitaires recherchent les outils susceptibles de les aider à bâtir au mieux leurs stratégies et à justifier ces dernières auprès des décideurs, en faisant notamment appel à des " modèles d'action de la communication ${ }^{1}$ » Ils sont ainsi amenés à mobiliser des concepts et notions le plus souvent importés des sciences humaines et sociales.

7 Mais si de nombreux écrits portent sur les campagnes publicitaires, ceux décrivant le processus de création et les modèles utilisés sont beaucoup plus anecdotiques. «Bien que la publicité soit omniprésente dans les sphères publique et privée et malgré l'abondante littérature qui lui est consacrée, peu de recherches scientifiques ont étudié la manière dont les messages naissent dans l'esprit des concepteurs. » (Courbet, Fourquet-Courbet, 2005, p. 67). Ainsi, qu'il s'agisse des réflexions menées par les professionnels, des méthodes employées, des tendances observées ou des résultats obtenus, les recherches scientifiques sur les pratiques $^{2}$ de création publicitaire sont rares. Aussi, la présente contribution, à visée exploratoire, tente-t-elle de mettre en évidence quelques-unes des logiques structurantes de ces pratiques. Elle s'appuie d'une part sur la littérature professionnelle existant sur le sujet (manuels, ouvrages monographiques, magazines professionnels depuis 2003), et d'autre part sur l'interrogation d'un échantillon ${ }^{3}$ de communicants (agences, annonceurs, services de conception intégrés), selon un mode d'investigation ouvert et semi directif. Les résultats de ces entretiens ont été traités par une analyse de contenu thématique.

8 Au travers de ces échanges, la performance de la création publicitaire apparaît comme une question récurrente. «La création publicitaire est un facteur essentiel de la performance de la campagne de publicité ; une bonne création permet de diminuer la quantité d'espaces achetés dans les médias tout en conservant une bonne efficacité grâce, entre autres, à la mémorisation qu'elle engendre; à l'inverse, une mauvaise création remet fortement en cause la performance de la publicité et peut dégrader l'image de la marque ou du produit concerné (alors qu'un mauvais plan média correspond simplement à un gaspillage économique du budget publicitaire). (Vernette, 2001, p. 103). Par ailleurs l'utilisation de modèles semble constituer l'un des fondements de la démarche créative.

Dans une première partie, nous nous intéresserons au processus de création publicitaire puis, dans une seconde partie, aux modèles mobilisés. Nous nous concentrerons sur les 
pratiques des créatifs, qu'ils soient concepteurs-rédacteurs (CR) auteurs des discours, ou directeurs artistiques (DA) concepteurs des images.

\section{La conception : un processus itératif fondé sur des théories incertaines}

10 La publicité peut être définie comme un processus de communication orienté émanant d'une organisation (entreprise, association, service public...) en vue de communiquer à un public donné un élément d'information (existence d'une offre ou d'un fait) par le biais de différents médias : presse, radio, télévision, cinéma, affichage, Internet. Cette publicité a beaucoup évolué depuis l'ancienne réclame. Aussi un bref historique mérite-t-il d'être rappelé avant de chercher à décrire le processus d'élaboration d'une campagne publicitaire.

\section{De L' « Unique Selling Proposition » à la communication multicanal}

Depuis la première partie du siècle dernier, des auteurs cherchent à théoriser les pratiques publicitaires. Ainsi, Claude Hopkins publie-il, en 1920, un ouvrage (Scientific Advertising) au propos ambitieux dans lequel il souhaite assimiler la publicité à une discipline scientifique, une science exacte, où il n'y a pas de place pour le doute. Il s'appuie sur les théories béhavioristes, un courant initié par Pavlov avec la publication de son ouvrage, Les Réflexes conditionnés, en1927. Durant plusieurs années, ces principes triomphent façonnant une génération de publicitaires pour qui le consommateurrécepteur du message est passif. Selon le modèle de "la seringue ", celui-ci n'est pas censé opposer de résistance aux messages qui s'inscrivent dans sa mémoire et influencent son comportement selon la fréquence de répétition. Cette conception publicitaire s'inscrit dans un marketing de masse proposant alors des produits indifférenciés à un large public, et s'appuyant sur les grands médias.

En cohérence avec cette vision simpliste, l'Unique Selling Proposition (USP) de R. Reeves (1961) domine les pratiques publicitaires durant de longues années et fait encore aujourd'hui de nombreux adeptes. Elle part du principe qu'une campagne doit formuler une proposition unique forte et la répéter selon la formule "repetition is persuasion ». Elle est à l'origine de nombreuses grandes campagnes qui ont traversé le temps, comme les fameux «Il fond dans la bouche et pas dans la main » de M \& M's, "Rafraîchit votre haleine pendant qu'il nettoie vos dents» (Colgate), ou le « Recommandé par les éleveurs » de Pedigree Pal.

Accusées, dans les années soixante, de conduire à une certaine uniformisation et tournées ensuite en dérision par des humoristes talentueux, dont le célèbre Coluche, les publicités doivent cependant évoluer au fil du temps en considérant les facteurs multiples et contradictoires de leur environnement du moment. Au cours de cette même période, le développement de la concurrence et d'une offre abondante et variée contribue à provoquer une diversification des attentes des consommateurs; toutes les recherches montrant qu'il n'est pas possible de parler d'un marché et d'une culture homogènes, même au plan national (Rutigliano, 1986). Aussi les positions s'affinent-elles au cours des années quatre-vingt avec l'interpénétration grandissante des marchés, de nouveaux modes d'organisation des entreprises, le développement de médias transnationaux et la 
constitution de réseaux planétaires d'agences de publicité. Les publicitaires se professionnalisent et se définissent, pour la première fois, comme des «techniciens » qui tentent de rationaliser leurs méthodes et d'adapter leurs pratiques aux réalités du marché. L'implantation d'agences américaines en France n'est pas étrangère à cette évolution; le créatif perdant peu à peu la latitude dont il disposait précédemment pour ne devenir qu'un maillon au sein d'une grande équipe évoluant au carrefour d'exigences variées (commerciales en particulier). Puis, en raison de la concurrence exacerbée existant entre marques (et/ou entreprises), de la complexité accrue de la demande, de la fragmentation des médias dits traditionnels (presse, radio, télévision...), mais également du sentiment de saturation ressenti par un nombre croissant de consommateurs face aux messages habituels, des doutes commencent à s'insinuer parmi les annonceurs vis-à-vis de ce type de communication. En outre, l'augmentation des tarifs publicitaires ainsi que l'encombrement croissant des supports médiatiques, amènent les organisations à envisager d'autres stratégies de communication (marketing direct par exemple), voire de nouveaux circuits de distribution de leurs produits. De même, l'avènement du WEB modifie-t-il profondément le paysage communicationnel en faisant apparaître de nouveaux acteurs qui obligent les marques traditionnelles à s'orienter vers des approches différenciées de leur politique commerciale. Confrontés à ces difficultés, la grande majorité des annonceurs en vient à utiliser alors plusieurs canaux de communication, de manière sélective, conjointe et complémentaire en s'orientant de plus en plus vers une communication intégrée fortement présente sur le Net avec possibilité de commerce en ligne. A titre d'exemple, nous citerons la saga publicitaire actuelle de la téléphonie Alice utilisant simultanément la télévision, la presse, la radio, l'affichage, le cinéma et Internet.

Mais l'intégration communicationnelle, qui consiste à utiliser en synergie les divers outils (presse, radio, affichage, $\mathrm{TICs}^{4} .$. ) et moyens de communication (relations publiques, promotion...) pour contribuer efficacement aux objectifs de l'entreprise, pose certaines difficultés. Elle nécessite, en effet, d'avoir une architecture communicationnelle agile, conviviale, susceptible de servir de façon cohérente les ventes à court terme tout en permettant de construire l'indispensable image à long terme... l'une pouvant difficilement être privilégiée au détriment de l'autre. La cohérence de l'ensemble des messages véhiculés devient alors un enjeu majeur, et non plus une simple question technique comme ce fut le cas auparavant. Aussi, dans cet univers marqué par une sophistication des moyens, l'influence de l'USP reste-t-elle vive. Elle participe au débat sur l'efficacité ${ }^{5}$ et inspirent des pratiques d'agences qui se concentrent sur la répétition de slogans faciles à mémoriser, au nom d'une publicité réaliste et efficace se concentrant sur les ventes. Toutefois, la gestion la communication ne résout en rien la question du message à faire passer... si ce n'est en complexifiant la situation avec des moyens et supports qui ne fonctionnent pas de la même façon.

Mais comment les publicitaires produisent-ils leurs messages et comment est conçu le contenu des campagnes?

\section{Des pratiques professionnelles itératives}

La conception d'une campagne de communication est habituellement fondée sur les grandes lignes d'une stratégie et d'un plan marketing définis préalablement, qui précisent les objectifs à atteindre. C'est le créatif (qu'il soit prestataire de service en agence ou intégré dans un service de l'annonceur) qui conçoit et rédige les messages 
publicitaires à partir d'un axe de campagne défini par les services commerciaux, en tenant compte des caractéristiques du produit et des médias retenus. Il élabore le message sur la base d'un brief, souvent centré sur une copy strategy ${ }^{6}$. Pour cela, il doit faire preuve de créativité, de capacité d'analyse et de synthèse afin d'être à même d'interpréter les données concernant le marché pour en tirer des arguments vendeurs. Ce faisant, il devient tour à tour technicien spécialisé, théoricien et créateur.

Nous reprendrons, ici, les différentes phases du processus telles qu'elles nous ont été exposées verbalement ou qu'elles sont indiquées dans les divers ouvrages professionnels. Tout d'abord, lors de la phase de conception, le concepteur, en collaboration avec le directeur artistique s'il existe, met au point plusieurs projets suivant les supports envisagés : pour les annonces et les affiches, un titre et une image, et pour les messages audiovisuels, un argument et un thème de film. La tâche du créatif est de rédiger les différents textes des messages publicitaires visuels (annonces, affiches) ou sonores (textes, communiqués radiophoniques, scénarii, dialogues de films et/ou des spots publicitaires). Il peut également écrire des communiqués de presse, des présentations d'articles, des catalogues, des accroches et des textes de publicité sur le lieu de vente (PLV). Il définit ensuite la mise en page des textes et de l'image dans le support définitivement retenu. A cette fin, les praticiens interrogés indiquent consulter régulièrement des ouvrages, banques de données, cédéroms susceptibles de les aider à trouver des idées. Ils compulsent systématiquement les magazines mis à leur disposition pour diversifier leurs sources d'information. Ils s'y reportent fréquemment pour se tenir au courant des dernières productions, « voir qu'est-ce que font les autres » et/ou " garder le contact ». La majorité d'entre eux lisent en moyenne plus de cinq magazines par mois. Par voie de conséquence, ils fréquentent avec assiduité les magazines professionnels ( $C B$ News, Stratégies...). Pour tous les acteurs interrogés, ces derniers sont considérés comme le vecteur d'une information permettant de les tenir au courant de l'actualité, de " développer leur créativité » et/ou d'affiner leurs connaissances du monde environnant. Or, il apparait que les articles de ces journaux spécialisés font fréquemment référence au même petit nombre de "modèles d'action de la communication ${ }^{7}$ " pour commenter les campagnes. Les termes de conditionnement et de répétition sont utilisés à de multiples reprises même si parfois certains s'en offusquent: «( .. ) l'implication dans un produit ne se formate pas par la répétition. À l'encontre de ces théories béhavioristes du conditionnement, cette approche met l'accent sur la diversité des modes d'interprétation de la communication et sur leur complexité. » (Stratégies, $n^{\circ} 1407,30 / 03 / 2006$, p. 6). De même, la notion de «besoin ${ }^{8}$ » estelle omniprésente. "Il est clair que la capacité de faire évoluer son métier pour accompagner les changements du marché et l'émergence de nouveaux besoins chez les clients est et restera toujours un facteur clé de succès. » (Revue des marques, 42, avril 2003, p. 31). D'autres références sont également mobilisées, comme la dimension rationnelle d'un acte d'achat. "Avec un nouveau logo, une nouvelle signature et une nouvelle campagne institutionnelle, l'assureur souhaite se différencier des bancassureurs, rappeler son positionnement du bon rapport qualitél prix et surtout qu'il est un généraliste. » (CB News, 22/06/2006, p. 15). La notion d'inconscient est aussi souvent reprise. "Un vent de contestation souffle, qui fait étrangement écho à l'actualité. La pub révèlerait-elle l'inconscient de notre société?" (Stratégies, n¹392, 01/12/2005, p. 21). Ces références diverses sont reprises par les créatifs eux-mêmes dans leurs discours, de façon plus ou moins mélangée, mariant les notions entre elles (besoin et inconscient par exemple) et sans toujours les différencier. Mais sont-elles mobilisés lors du processus de création? 

les praticiens de la publicité oscillent entre deux stratégies ; ces deux conceptions n'étant pas exclusives, mais se complétant, se confrontant, s'enrichissant mutuellement (Snyder, Bono de, 1985). "Tantôt ils font préférentiellement appel au rôle des symboles associés à l'usage des produits, tantôt ils se focalisent sur les qualités inhérentes aux produits ou sur leur valeur fonctionnelle. Ainsi les auteurs distinguent-ils les publicités fondées sur l'image de ce que représente le produit, des publicités fondées sur l'argumentation intrinsèque, même s'il est vrai que dans la pratique ces deux stratégies sont souvent liées et dépendent des types de produits.» (Georget, 2004, p. 73). que par des théories'. Ils indiquent appliquer à chaque situation de création particulière des «modèles d'action de la communication » élémentaires, à leurs yeux " pragmatiques »: ils les connaissent, les considèrent comme des «moyens » mis à leur disposition, estiment qu'ils présentent un caractère universel, quels que soient les situations, les annonceurs et/ou les individus ciblés.

ombreux sont les créatifs qui rappellent d'emblée qu'un individu est en contact avec de très nombreux messages chaque jour, dont seule une vingtaine est perçue et moins d'une dizaine influencent son comportement. «Le consommateur moyen est confronté chaque jour à trois mille messages publicitaires. La perception, à $70 \%$, se fait de façon non-verbale, alors que les études s'appuient sur une restitution verbale de type "Avez-vous vu... ». Une bonne mesure de l'efficacité doit être capable de prendre en compte cette dimension. "(Stratégies, $\mathrm{n}^{\circ} 1418$, 15/06/2006, p. 22). Les publicitaires apparaissent ainsi démunis devant des demandes de résultats toujours plus exigeantes. Pour la plupart, ils soulignent la difficulté croissante du travail de création publicitaire et indiquent l'importance prise par l'auto-évaluation de la qualité de leur production. A chaque étape, le concepteur juge son message, le critique, opère des modifications, en compare les états, alterne phases de conception et phases d'évaluation. Il confronte plusieurs projets suivant les supports envisagés, les amende et définit la mise en page des textes et de l'image dans le support définitivement retenu, en accord avec ses collègues. Le processus d'évaluation se révèle ainsi itératif, tantôt individuel, tantôt collectif, avec de nombreuses discussions, avancées, retours en arrière et jugements intermédiaires.

Ce n'est que lorsque l'équipe créative estime que sa production est suffisamment proche de ce qu'elle pense être la volonté du prescripteur qu'elle arrête le processus de conception. A cette fin, les publicitaires cherchent systématiquement à connaître les critères explicites et implicites qui seront utilisés par leurs clients pour juger les projets qui leur seront soumis ; l'objectif étant de construire une campagne qui répondra à leurs critères et d'incorporer ces derniers dans l'argumentaire de présentation du message finalisé. L'équipe de création souhaite ainsi pouvoir justifier au mieux ses choix en épousant les critères d'évaluation et d'acceptation du décideur. Nous retrouvons ici les résultats de recherches récentes concernant la publicité sur Internet. "Le concepteur ne cherche pas systématiquement à concevoir les messages les plus influents, mais à créer des messages dont la justification est à même d'être acceptée par l'annonceur. Dans ce cas, cette approche satisfaisante de la production nécessite une justification a posteriori du processus de conception. L'objectif est de donner au message l'apparence de la rationalité et notamment de la conformité aux règles de création publicitaire classiquement utilisées dans la profession. L'argumentation que le publicitaire va développer face à l'annonceur pour "vendre » sa bannière doit être perçue comme suffisamment persuasive pour convaincre l'annonceur que la bannière va 
effectivement réaliser ses objectifs " (Courbet, 2005, p. 73). Bien que le recours aux modèles soit jugé fort "utile ", notamment pour bâtir l'argumentaire utilisé pour convaincre les décideurs, les professionnels interrogés semblent ainsi suivre, le plus souvent, une démarche aléatoire, itérative et parfois erratique !

De plus, ils ne semblent pas tous maîtriser de la même manière les modèles auxquels ils se réfèrent. Les résultats de notre enquête montrent que leur connaissance est souvent mal assurée ; plus de la moitié des personnes interrogées estimant qu'elles n'ont pas besoin d'en connaître précisément les fondements théoriques sous-jacents. Ils peuvent citer quelques éléments des théories qui orientent leur démarche à chaque étape du processus de conception, mais sans toutefois les restituer dans leur intégralité, sans citer leurs auteurs, leur domaine d'application ou leurs limites respectives. Ils considèrent, que la « communication n'est pas une science exacte » et ne se limite pas à un seul mode d'analyse. Les «théories ", les « modèles » sont envisagées comme pouvant aider à mieux saisir la « réalité du moment ", «l'air du temps » afin de trouver ce message percutant susceptible de toucher la cible visée. Ils peuvent certes contribuer à « stimuler la créativité » mais servent surtout " comme arguments " pour valider les productions réalisées auprès de leurs interlocuteurs.

Ainsi, même si la création publicitaire est présentée par une majorité de professionnels comme un processus de prise de décision issu d'une démarche rationnelle (Cohen, March, Olsen, 1972 ; March, 1978 ; Simon, 1983 ), fondée sur le respect de la copie stratégie, la formalisation du problème, la recherche et la sélection de la meilleure solution (Simon, 1983), elle intervient plutôt comme une suite d'"actions situées"10, prises par les créatifs en réaction à un contexte mouvant sans application d'un plan préétabli (Suchman, 1987; Hutchins, 1991, Weick, 1995), et faisant l'objet de justification a posteriori (March, 1978). Le caractère partiel et précaire des consensus élaborés entre les divers intervenants conduit à concevoir leurs représentations collectives non comme un phénomène durable mais comme des artefacts sociaux transitoires (Langfield Smith, 1992), c'est-à-dire des points d'accord temporaires, négociés par les membres du groupe en interaction ; en outre, la première solution jugée satisfaisante est généralement retenue sans véritable recherche d'optimisation.

24 Après cette tentative de compréhension du processus de conception publicitaire, il nous reste à aborder la représentation des acteurs en présence et notamment la nature des différents modèles mobilisés.

\section{Du bricolage au braconnage}

25 Au delà des rappels sur le modèle bien connu de Shannon et Weaver, plusieurs références théoriques semblent s'affronter dans les discours des publicitaires. De même, il existe une abondance de références personnelles utilisées comme modèles d'aide à la création, liées à la fois à la formation personnelle des créatifs et à la variété de leurs itinéraires. Nous pouvons cependant identifier des éléments de convergences dans cette profusion apparente. 


\section{Pluralité des modèles} schématiquement à un petit nombre de références, plus ou moins imbriquées les uns aux autres.

\section{La pyramide des besoins}

Abraham Maslow a défini une hiérarchisation des besoins humains, sous forme de pyramide, dans le but de définir les leviers de la motivation. Son raisonnement est fondé sur le fait que l'individu a des besoins hiérarchisés; les besoins d'ordre supérieur n'étant ressentis par l'individu que lorsque les besoins d'ordre inférieur sont satisfaits. Sa pyramide part des besoins physiologiques, primaires de survie, tels que nourriture, eau, air, sommeil. Vient ensuite le besoin de sécurité physique (vêtements, toit, foyer...) puis les besoins sociaux ou de reconnaissance. Le besoin d'estime correspond au besoin de signes d'appréciation positifs émis par les autres. La réalisation de soi est l'affirmation de son individualité, ce qui identifie l'individu et le rend unique.

Tous les publicitaires interrogés jugent nécessaire d'adapter les messages aux différents marchés en se basant sur les besoins ${ }^{11}$ à satisfaire. "Chaque message doit répondre à un besoin identifié $d u$ consommateur » précise l'un de nos interlocuteurs.

Présentant l'avantage de pouvoir être aisément mémorisée ${ }^{12}$, la pyramide de Maslow est systématiquement invoquée comme fondement initial de la conception. Les magazines professionnels s'y réfèrent fréquemment, estimant que tout achat de produit «correspond à l'état de maturité de notre société où chacun a besoin de se réaliser (...) Ce comportement renvoie à la pyramide de Maslow, du nom de son inventeur, le psychologue américain Abraham Maslow, qui s'est penché sur les besoins et les motivations de l'homme. Il distingue ainsi cinq étapes. À la base de la pyramide, la satisfaction de besoins primaires comme la nourriture. Et, à la pointe, le besoin de se réaliser. Pour passer de l'un à l'autre, l'homme recherchera d'abord la sécurité, ensuite à appartenir à un groupe, puis l'estime de soi. Pour se réaliser, il suffit donc d'acheter un appareil photo numérique. Tout simplement. Ou presque... » (Stratégies, 1320, 01/04/2004, p. 32)

De nombreuses campagnes publicitaires s'en inspirent directement, telle que celle d'Alice déjà citée, dans laquelle la jeune femme, qui symbolise la marque, répond directement à des personnes apparemment insatisfaites en termes de "réponse à leurs besoins ».

Notons toutefois que cette hiérarchisation est jugée critiquable par quelques concepteurs (six d'entre eux) pour son caractère réducteur. Les besoins sont alors considérés "variables d'un individu à l'autre », "l'intensité des besoins est répartie de manière différente ", selon les différentes phases de la vie (études, vie en couple, arrivée d'un enfant, retraite...) qui «influencent leur hiérarchisation». En outre, selon les circonstances, des besoins supérieurs peuvent primer les besoins élémentaires, par exemple « en situation extrême ».

\section{Le modèle de l'homo economicus}

En contrepoint de la pyramide de Maslow, les publicitaires citent "la théorie économique classique » avec l'homos economicus. L'individu est alors considéré comme un être logique au comportement dicté par la raison et le calcul. Ses achats et sa consommation 
s'expliquent par l'utilité d'un produit, c'est-à-dire sa valeur d'usage. "Une étude de TNS Media Intelligence montre un retour vers la réclame d'antan, avec des communications axées sur les prix.» (Stratégies, 1356, 10/02/2005, p. 43)

La tendance à mobiliser cette facette de l'individu semble privilégiée depuis quelques années. «Dans un climat socioculturel déstabilisant, en plein débat sur le sens de la consommation et dans un contexte global de réflexion " antimarque ", le discours prix a le mérite d'appeler un chat un chat. On voit également apparaitre l'idée de consommer malin, parallèlement au développement des compagnies à bas prix et du " hard discount " (Stratégies, 1356, 10/02/2005, p. 43). Les exemples abondent dans les campagnes publicitaires actuelles, notamment celles des distributeurs (Intermarché, Leclerc, Carrefour...). Sous forme d'une bande annonce de film hollywoodien, un marchand de meubles retrace, par exemple, l'histoire d'un amour impossible entre une «belle chaise et un petit prix », et leur « fuite » éperdue vers l'univers Ikea. De même, le spectateur peut-il regarder à la télévision les douze secondes de l'eau Cristalline, valorisée par l'entraîneur Guy Roux, connu pour sa parcimonie, ou écouter à la radio la publicité pour les voitures Logan de chez Renault dont le prix est mis en exergue.

Ce modèle est également utilisé à l'international. Même des marques cosmétiques, censées vendre du rêve, s'y mettent. Ainsi la marque Olay a récemment publié une annonce aux Etats-Unis indiquant explicitement que sa crème antirides est moins chère et plus efficace que celles de ses concurrentes. Même si toutes ne prennent pas cette direction, certaines font également appel à la rationalité du consommateur comme l'Oréal, par exemple, qui tente de fournir la preuve, schéma millimétré à l'appui, de l'efficacité de ses produits sur les visages de femmes célèbres.

\section{Le modèle sociologique}

Les notions de base le plus souvent citées en référence à ce modèle sont celles de groupe social, de norme, de statut dans le groupe et de leader d'opinion. Le type de publicité correspondante est qualifié d'intégrateur car il joue sur le «besoin» du consommateur à faire partie d'un groupe. Selon le modèle du two step flow of communication de Katz et Lazarsfeld (1955), il est plus efficace et rentable d'orienter l'action publicitaire vers un nombre limité de leaders qui transmettent, ensuite, le message à leur cercle de connaissances. De même, des contenus publicitaires montrent des "stars" issues de groupes sociaux particuliers, afin que, par identification, le produit et la marque deviennent l'élément fédérateur du groupe, un signe de réussite et de reconnaissance sociales. Les "styles de vie » de Cathelat y font également référence et, même s'ils ne bénéficient plus de l'engouement passé, sont encore cités (par un cinquième des créatifs interrogés).

Ce modèle a été récemment utilisé par la société Nike. Des enquêteurs ont passés les banlieues au crible pour identifier les virtuoses du basket ou du football, susceptibles d'imposer une mode à leur entourage, voire à une classe d'âge. «Les " advocates " ou défenseurs de la marque, le coeur de cible des sportifs pratiquants réguliers, (...) véritables inconditionnels capables de transmettre la bonne parole de la marque, sont des leaders d'opinion pour Nike. L'adoption d'une nouvelle paire de chaussures par cette communauté a toutes les chances de convertir une classe d'âge. Nike est à même d'identifier la centaine de ces athlètes du quotidien en envoyant ses enquêteurs dans les banlieues, et organise pour eux des opérations de 
street-marketing spécifiques : distribution de flyers, de T-shirts, de CD-Rom, etc. "(Stratégies $\mathrm{n}^{\circ}$ 1289 du 11/07/2003, p. 46)

De même, la firme Coca-Cola a-t-elle recruté une ancienne miss France devenue présentatrice $\mathrm{TV}$, pour livrer des conseils aux téléspectatrices lors de la dernière coupe du monde de football. "Nouer un partenariat avec une vedette peut donner un sérieux coup d'accélérateur à une opération de communication. Chacun y trouve son compte : la marque bénéficie d'un porte-parole idéal, la star d'une campagne de promotion à grande échelle. (...) Le recours aux vedettes, leaders d'opinion d'opportunité, est un moyen pour une marque de s'assurer des retombées presse. Il permet aussi à la célébrité de valoriser son actualité. » (Stratégies n 1289 du 11/07/2003, p. 46).

\section{Le modèle du conditionnement}

Ce modèle est fondé sur l'expérience du « chien de Pavlov » qui salive au simple son d'une cloche parce qu'il anticipe le morceau de viande juteux qui suivra ${ }^{13}$. Il laisse supposer que la persuasion est le résultat de la répétition et qu'il suffit de répéter un nom de marque pour que la cible la retienne et la réclame. Sachant que chaque individu ne peut retenir que deux ou trois noms de marques dans une catégorie de produits donnée, il s'agit d'amener ce nom en tête de liste, top of mind, dans la tête des cibles visées car c'est celle qui a le plus de chance d'être choisie au moment où la cible prendra une décision d'achat.

Les produits visant un large public, et surtout ceux à faible coût dont l'achat est impulsif et répond à un besoin cyclique, recourent à cette approche. C'est le cas, par exemple, des boissons gazeuses ou des loteries. Ainsi, Coca-Cola, voyant ses marchés décliner, a annoncé au printemps 2001 qu'il augmentait ses budgets publicitaires de près de $50 \%$.

Fréquemment citée par les personnes interrogées, les manuels et ouvrages de marketing, la méthode «AIDA $»^{14}$ qui vise à attirer l'attention du prospect à tout prix à l'aide d'éléments souvent sans lien avec les caractéristiques du produit, renvoie également à ce modèle.

41 Le modèle du conditionnement est repris dans le camp de la critique sociale de la publicité; la pensée antipub y trouvant le principal ressort du marketing et de la communication : «La publicité s'attaque à notre cerveau (...). Elle nous conditionne.» écrivent les Casseurs de pub ${ }^{15}$.

\section{Le modèle psychanalytique}

42 Si le béhavioriste assume que l'humain réagit à son environnement, Freud explique dans Introduction à la psychanalyse (1929) qu'il agit plutôt en fonction de forces internes obscures, les instincts, oscillant entre Éros (l'instinct de survie) et Thanatos (l'instinct de destruction).

Les tenants du modèle psychanalytique en publicité partent du principe que les consommateurs ne sont pas conscients des motivations qui gouvernent leurs choix car elles sont à rechercher dans le ça, qui renferme leurs pulsions profondes. Le rôle du publicitaire est d'identifier puis de flatter ces désirs profonds tout en bravant les interdits du surmoi. Seul l'acte d'achat permettrait de résoudre le conflit psychique entre le désir et l'interdit qui est source d'angoisse. Aussi, des arguments sont-ils proposés à l'individu pour rationaliser ses choix et les justifier de manière socialement acceptable. Pour les 
publicitaires, les objets se vendent en effet bien davantage en mettant en évidence leur " valeur symbolique » qu'en argumentant sur leur valeur d'usage. Le produit vient ainsi combler un manque affectif ou narcissique permettant à l'individu d'accéder au bonheur et à l'épanouissement personnel.

La publicité joue sur ce désir comme les publicités de l'Oréal et son slogan « parce que je le vaux bien" qui flattent et rassurent le consommateur. En affichant des personnages auxquels souhaiteraient ressembler les consommateurs, la publicité offre à ces derniers des moyens d'identification au travers d'une abondance de produits. De même, les messages à connotations sexuelles foisonnent-ils, utilisant des stimuli érotiques explicites ou cachés, le plus souvent sans lien direct avec le produit (cf le phénomène du " pornochic » des marques de luxe, les mises en scène du café...nommé "désir " ou celles de certaines boissons alcoolisées).

\section{La dissonance cognitive}

Une mention particulière doit être réservée au modèle de la « dissonance cognitive » de Léon Festinger (Theory of Cognitive Dissonance, 1957), qui semble bénéficier actuellement d'un phénomène de mode.

D'après ce modèle, chaque individu se comporte selon l'équilibre interne qu'il s'est créé et la communication de nouvelles informations peut le déséquilibrer. Afin de rétablir un nouvel équilibre interne, l'individu évalue cette information pour l'intégrer dans son univers. Quand il se retrouve en situation de dissonance cognitive, il peut diminuer celleci en modifiant son comportement, tenter d'atténuer la portée de l'information déséquilibrante ou rechercher une information contredisant celle-ci.

Le modèle de la dissonance cognitive «montre que la cible réagit selon son expérience qu'elle a $d u$ produit ». Plus son implication est forte et moins elle souhaite remettre en cause ses choix. Elle tend ainsi à éviter ou filtrer les messages qui les contredisent et se révèle réceptive à ceux qui les confortent. Ce modèle explique notamment ce que les publicitaires appellent l'« exposition sélective » : les consommateurs «ne voient pas» les annonces qui peuvent trop gravement compromettre leur équilibre interne et évitent même les circonstances qui pourraient les confronter à de l'information déséquilibrante. "Il n'y a rien de plus difficile que de convertir un utilisateur d'Apple à un PC. », de même "Celui qui a une Mercedes ne voudra jamais reconnaittre la supériorité d'une BMW». Toutefois, certains publicitaires recommandent aux marques de poursuivre la pression publicitaire bien après la phase d'achat afin d'éviter que les acheteurs ne soient mis dans un état d'inconfort psychologique par des publicités de produits concurrents.

Malgré tout, certaines équipes créatives cherchent à créer une telle dissonance, un malaise chez les destinataires, afin de se singulariser tout en espérant obtenir l'adhésion d'une partie du public ${ }^{16}$. A l'époque de nos entretiens, la campagne publicitaire de la collection automne-hiver 2006-2007 de la marque de vêtements Marithé et François Girbaud faisait beaucoup parler d'elle. Axés sur le thème de la guerre, les visuels mettaient en scène des femmes et des hommes jeunes couverts de boue et de poudre à canon dans un paysage dévasté.

Les modèles communicationnels utilisés ne sont pas homogènes et semblent mobiliser différents paradigmes, références théoriques et méthodes. Les concepts retenus sont des termes polysémiques, pouvant être qualifiés de nomades (au sens de Stengers). Ils ont 
migré d'un champ scientifique à l'autre, chacun de ces derniers ayant contribué à les définir avant d'être utilisé à leur tour par les publicitaires. Ils nous incitent à rappeler que les liens entre les sciences humaines et sociales (SHS) et la publicité sont étroits. En son temps, Watson, vice-président de l'Association américaine de psychologie, n'a-t-il pas abandonné l'Université pour devenir directeur général de l'agence J. Walter Thompson? Ainsi des scientifiques se penchent-ils sur la question afin d'identifier des modèles «mécanistes » qui dévoileraient des moyens de persuasion parfaitement efficaces, voire machinaux. De même, le laboratoire de neuropsychologie Mind of the Market, rattaché à la célèbre Harvard Business School, cherche-t-il, depuis plusieurs années, à savoir précisément comment la publicité agit sur le cerveau des consommateurs au moment de l'achat. " Notre intention n'est pas de manipuler les goûts de nos semblables, affirme sans ciller le professeur de neuropsychologie, Stephen Kosslyn, nous sommes simplement les premiers à mettre au service des entreprises toutes les connaissances fondamentales sur le rôle des zones cérébrales dans les émotions et le comportement. Notre travail encore très préliminaire, devrait à terme changer radicalement les techniques de marketing. » (Sciences et Avenir, septembre 1999, p. 26).

\section{De la profusion à la confusion}

Les modèles et références précédemment cités sont repris abondamment par les créatifs dans leurs discours, mais de façon plus ou moins mélangée, mariant les notions entre elles sans toujours les différencier. Ces concepts incertains se chargent selon les acteurs concernés de divers traits sémantiques donnant lieu à des sens différents, sans se départir pour certains de leur ambiguïté originelle (cf. besoin). Il apparaissent pour la plupart schématiques et datés pour expliquer des réalités complexes; la persuasion publicitaire ne semblant pas aussi simple qu'ils le laissent entendre. De plus, les recommandations découlant des diverses approches avancées, que ce soit pour persuader le prospect ou pour capter son attention et laisser une trace durable dans sa mémoire, sont parfois contradictoires entre elles. En conséquence, les allégations correspondantes sur les effets de la publicité paraissent bien aléatoires!

51 De même, les termes utilisés, que ce soit pour les slogans ou les noms de marque, ne respectent pas toujours leur définition originale. Reprenons l'exemple du mot « zen » (cf. "un placement financier pour rester zen", "restez zen grâce aux flèches FNAC", un thé qui produit un "corps zen, esprit zen»,) dont l'utilisation poussant à la consommation va à l'encontre de leur signification première relative aux valeurs du bouddhisme : frugalité, ascèse, maîtrise du désir.

Par ailleurs, le respect de la réglementation et de la déontologie est jugée très, voire trop, « contraignante » par les personnes interrogées. Certaines mentions ${ }^{17}$ ayant été éliminées des propositions créatives, car jugées abusives par le BVP ${ }^{18}$ ou la DGCCRF ${ }^{19}$, suscitent de vives réactions. Ces limitations, vivement critiquées, sont considérées comme des " entraves à la créativité».

Aussi, l'étude des pratiques laisse apparaître que les publicitaires semblent parfois hésiter entre le bricolage (Lévi-Strauss, 1922), activité improvisée adaptée aux circonstances, et le braconnage (De Certeau, 1980) qui recouvre la reprise et le détournement d'idées venues d'ailleurs, le respect relatif des droits ou de la déontologie et la justification a posteriori, au coup par coup, variant selon les interlocuteurs. Contraints à l'obtention 
rapide de résultats dans un environnement très concurrentiel, devant justifier leur activité et assurer leur avenir, les communicants s'estiment toutefois dans l'obligation de recourir à de telles solutions, faites de bricolage ou de braconnage, pour créer des publicités, mesurer leurs performances et convaincre leur interlocuteurs au moyen d'un appareillage théorique incertain.

\section{Conclusion} mobilise le plus souvent des pratiques collectives enrichies du fruit des expériences passées sous forme de techniques et objets. La notion de traduction (au sens de Callon et Latour) est au coeur de ce processus de conception : les acteurs travaillant constamment à traduire leurs problèmes, langages et intérêts dans ceux des autres.

A heure du débat sur l'efficacité, le recours aux modèles d'action de la communication devrait permettre de mieux comprendre les différents chemins qu'empruntent les créatifs pour toucher la cible visée. Mais conçu comme un instrument de travail par les intéressés eux-mêmes, le modèle n'apparaît pas immuable et semble diversement utilisé tout au long de la recherche créative. Employé d'abord pour soi, afin de faire émerger les premières idées, il est ensuite enrichi, affiné ou détourné selon les sollicitations des acteurs en présence et ce, au gré des circonstances. C'est à travers lui que la stratégie est vérifiée, que les moyens à mettre en place sont évalués, que l'approche retenue est jugée pertinente. Il sert notamment, par la suite, à rassurer ou convaincre les clients, au moment de l'évaluation de la proposition créative. Ainsi, l'étude des pratiques montre que les publicitaires hésitent entre bricolage, utilisation incertaine des divers modèles d'action de la communication dans le processus créatif, et braconnage,pratiqueadaptée aux circonstances dans lequelle respect des règles déontologiques est relatif.

En dépit des travaux menés par certains chercheurs et professionnels de la communication actuellement, la modélisation fine du comportement des consommateurs et de leurs réactions à divers stimuli provoqués nous apparaît encore bien lointaine. Aussi ne pouvons-nous que nous réjouir de cette conservation d'une part d'autonomie des individus, même si l'influence de la publicité sur l'évolution des sociétés apparaît indéniable.

\section{BIBLIOGRAPHIE}

Baffoy T., Burnet R., « Dieux et usages de dieux : OPA publicitaire sur le sacré », Esprit, 8-9, aoûtseptembre 2006, p. 16-29.

Certeau M. De, L'invention du quotidien, tome 1 : Arts de faire. Paris, UGE, 1980.

Cohen M.D., March J.G., Olsen J.P., “A garbage can model of organizational choice”, Administrative Science Quaterly, 1972, 17, p. 1-25.

Communication et organisation, $30 \mid 2006$ 
Courbet D., Fourquet-Courbet M-P., « Les processus psychologiques lors de la création publicitaire ", Hermès, 2005, 41, p. 67-74.

Courbet D., « L'influence non-consciente des publicités vues furtivement et aussitôt oubliées. Une méthode d'étude sociocognitive appliquée à l'Internet », Questions de communications, 2004, 5, p. 83-102.

Courbet D., « Les applications des sciences humaines à la publicité : De la psychanalyse à la sociocognition implicite et au neuromarketing ", Humanisme et Entreprise, 2006, n² 276, p. 1-20

Delcambre P., et al., Communications organisationnelles, objets, pratiques, dispositifs. Rennes, Presses Universitaires de Rennes, 2000.

Erdelyi M. H., Zizak D.M., Beyond G, “Subliminality”, In Schrum L.J., (ed.), The Psychology of Entertainment Media. Mahwah (New Jersey) Lawrence Erlbaum Associates, 2004, p. 13-43

Festinger L., A theory of Cognitive Dissonance. Evanston, Row, Perterson, 1957.

Fourquet-Courbet M-P., « Influence attendue et influence effective de la publicité sur l'Internet. Des représentations sociales des producteurs aux modèles scientifiques » Questions de communications, 2004, 5, p. 31-55.

Georget P., « Effets des stratégies publicitaires. Personnalité, contexte et attentes contractuelles », Questions de communications, 2004, 5, p. 69-83.

Hutchins E., "The social organization of distribued cognition. In Resnick L.B., Levine J.M., Teasley S.D.", Perspectives on socially shared cognition. Washington, DC, American Psychological Association, 1991, p. 283-307.

Joannis H., Barnier de V., De la stratégie marketing à la création publicitaire. Paris, Dunod, 1995 Jouet J., « Retour critique sur la sociologie des usages », Réseaux, 2000, nº 100, p. 489-521. Langfield-Smith K., "Exploring the Need for a Shared Cognitive Map", Journal of Management Studies, 1992, n²9 (3), p. 349-368.

Lebel P., Métrologie, mesure et maîtrise des communications. Paris, Editions ESF, 1980.

Leleu-Merviel S., La conception en communication, méthodologie qualité. Lille, Hermès Sciences Publications, 1997.

Lendrevie J., Baynast de A., Publicitor. Paris, Dalloz, 2004.

Marcenac L., Milon A., Saint-Michel S.-H., Stratégies publicitaires. Paris, Bréal, 2002.

March J.G., Décisions et organisations. Paris, Editions d'organisation, 1978.

Radu M., « De la comparaison sociale à l'intention comportementale. Les publicités pour produits cosmétiques amincissants ", Questions de communications, 2004, 5, p. 103-114.

Rutigliano A.J., "The debates goes on : global vs local advertising”, Management Review, 1986, p. 35.

Simon H.A., Administration et processus de décision. Paris, Economica, 1983.

Suchman L., Plans and situated actions: The problem of human-machine communication. New York, Cambridge University Press, 1987.

Synder M., Bono K.G. de, "Appeals to image and claims about quality : understanding the psychology of advertising”, Journal of Personality and Social Pyschology, 1985, 49, p. 586-597.

Vernette E., Publicité. Théories, acteurs et méthodes. Paris, La documentation française, 2000. 
Weick K.E., Sensemaking in Organizations. Sage Publications, Fondations for Organizational Science, Thousand Oaks, CA, 1995.

\section{NOTES}

1. Terme utilisé dans les ouvrages professionnels et que nous reprendrons tel quel dans cet article, mais dont la définition mériterait d'être interrogée.

2. Nous reprendrons ici la définition de la pratique proposée par Jouët (1993, p. 371) dans un article de référence, à savoir: "une notion plus élaborée qui recouvre non seulement l'emploi des techniques (l'usage) mais aussi les comportements, les attitudes et les représentations des individus qui se rapportent directement ou indirectement à l'outil »

3. Largement utilisé, ce terme d'échantillon pourrait prêter à confusion car il est le plus souvent associé à une démarche quantitative fondée sur un questionnaire standardisé. Or ce n'est pas le cas ici, compte tenu de notre approche qualitative.

4. Technologies d'information et de communication

5. L'évaluation de l'efficacité d'une campagne nécessite de définir précisément les critères d'évaluation à retenir, de mesurer ensuite les résultats obtenus au regard de ces critères préétablis, d'éliminer les hypothèses explicatives rivales (facteurs autres que la campagne comme les autres variables d'action marketing) et enfin de mesurer la productivité d'une campagne, c'est-à-dire ce qu'elle a rapporté à l'organisation concernée.

6. Appelée aussi plan de travail créatif, ou charte créative, ou star stratégie, ces préalables à la création publicitaire présentent des variantes (permettant notamment de différencier des agences concurrentes) mais cherchent à atteindre le même but : contribuer à l'émergence d'une campagne performante dotée d'une création attractive répondant aux objectifs marketing et publicitaires de l'annonceur.

7. présentés dans les nombreux ouvrages professionnels (Publicitor, Mercator, Pentacom, glossaires, dictionnaires...)

8. Nous reviendrons sur ce terme de besoin utilisé par les publicitaires interrogés plus loin.

9. Confirmant en cela les travaux portant sur l' "influence attendue et influence effective de la publicité sur l'Internet » (Fourquet-Courbet, 2004).

10. Ce terme d'action située a été introduit par L. Suchman, anthropologue, pour indiquer que l'action est orientée vers un but et dépend des moyens et des circonstances du moment. Il désigne le mode de l'action propre à l'ethnométhodologie, où «la situation d'une action peut être définie comme l'ensemble des ressources disponibles pour véhiculer la signification de ses actions et pour interpréter celles d'autrui. » (Suchman, 1987, p. 6). Elle se fonde sur l'observation des interactions quotidiennes, et considère que le sens de l'action sociale est produit dans le cours de l'action ellemême.

11. Le terme de besoin ici utilisé ne fait pas référence au concept scientifique (construit social ou invariant) correspondant, ni à ses limites, auquel ne se sont pas référés les publicitaires interrogés.

12. Largement reprise dans les manuels.

13. Cf. Pavlov et son ouvrage célèbre portant sur Les Réflexes conditionnés.

14. AIDA : attirer l'Attention, susciter l'Intérêt, créer le Désir, convaincre d'Acheter.

15. Site Les casseurs de pub, consulté le 16/08/2006.

16. Partant du principe que plus le spectateur d'une campagne est malmené par elle, plus il est tenté d'emprunter le chemin qui lui est indiqué pour résoudre son malaise. Cf la stratégie utilisée par la marque "Benetton " il y a quelques années. 
17. Par exemple, des allégations comme " maison », " fermier », " image d'une gousse de vanille »... donnant une image traditionnelle ou naturelle à un produit industriel

18. Association de professionnels pour une publicité responsable

19. Au sein du ministère de l'Économie, des Finances et de l'Industrie, la Direction Générale de la Concurrence, de la Consommation et de la Répression des fraudes, détecte et sanctionne les pratiques préjudiciables aux consommateurs (publicités mensongères, faux rabais, abus de faiblesse...) et vérifie la bonne application de la réglementation.

\section{RÉSUMÉS}

Longtemps considérée comme l'activité privilégiée du marketing, au point d'en être parfois confondue avec lui, la publicité cherche à obtenir un effet sur le comportement des individus d'une cible visée, en faveur d'un annonceur donné ; l'activité publicitaire pouvant difficilement s'envisager indépendamment de ses retombées. Dans le difficile contexte actuel, les publicitaires recherchent les outils susceptibles de les aider à bâtir au mieux leurs stratégies et à justifier ces dernières auprès des décideurs, en faisant notamment appel à des «modèles d'action de la communication ». Mais ces modèles n'apparaissent pas immuables et semblent diversement utilisés tout au long de la recherche créative. Employés d'abord pour soi, afin de faire émerger les premières idées, ils sont enrichis, affinés ou détournés selon les sollicitations des acteurs en présence et ce, au gré des circonstances. La présente contribution, à visée exploratoire, tente de mettre en évidence quelques-unes des logiques structurantes de ces pratiques. Elle montre ainsi que les publicitaires hésitent entre le "bricolage ", utilisation incertaine des divers modèles d'action de la communication dans le processus créatif, et le «braconnage » adapté aux circonstances, dans lequelle respect des règles déontologiques est relatif.

A study of the practices shows that publicists seem to hesitate between mending an improvised activity adapted to the circumstances but legal, and poaching in which the respect of the law is a very relative thing. Within the frame of their professional activity, the people in charge of communication are also trying to reach plurial goals. The present contribution, whose aim is exploratory, is based on the study of communication strategies over the last few years. It seeks to highlight some of the logics behind the professional practices concerned. It relies on a critical analysis of the theories and techniques developed by publicists.

\section{INDEX}

Mots-clés : publicité, modèle d'action de la communication, création, stratégie, évaluation

\section{AUTEUR}

\section{ARLETTE BOUZON}

Arlette Bouzon est Professeur en Sciences de l'Information et de la Communication à l'université Toulouse III, laboratoire ERITA-CERTOP Toulouse II. Mail : arlette.bouzon@iut-tlse3.fr 